\title{
Plasma Heart Failure Markers in Athletic Heart Progress in Long Distance Runners.
}

\section{Ayat Y. Bagato}

Lecturer in Biological sciences and sports health department, Faculty of physical education for girls, Helwan University, Egypt.

\section{Mohamed E.Dseouey}

Ass.professor in sports health department, Faculty of physical education for boys, Helwan University, Egypt.

\begin{abstract}
Background: Nature of the athlete's heart is still doubtful whether it is only a physiological adaptation or a potentially pathological condition. It is characterized by cardiac enlargement with Brady-arrhythmias in individuals participate in long distance runners with exercise capacity without attendant signs of cardiovascular disease .

Methods: Two different groups (each of eight subjects) chosen from the qualifying cross country race for Africa competition held on 24/2/2012 in Al Gizera youth centre. First group was adult and ran $12 \mathrm{~km}$. and the second was under 18 years and ran $8 \mathrm{~km}$. Both groups volunteered for this study. Their blood samples were collected pre and post race for assessment of cardiac markers NTproBNP, Galectin-3 and cTnI.
\end{abstract}

Results: All cardiac markers were elevated post exercise compared to that achieved in pre exercise regardless age of athletes. Also, pre exercise adult results were higher in galectin-3 and cTnI but not in proBNP while there is no difference in the acute response in both groups. Theses results revealed micro cardiac necrosis due to long run exercise with possible bad prognosis.

Conclusions: it is important to develop risk prediction and screening methods for athletic heart and consider it as disorder not physiological adaptation .

Key words: Brady-arrhythmias, athletic heart, Galectin-3, proBNP, cTnI, long distance runners, exercise capacity, cardiovascular disease.

\section{Introduction:}

Participation in endurance competitive
sport and/or vigorous recreational exercise like long run more than 10,000 meters and marathon races continues to gain in popularity all over the world. Documented health benefits of regular physical exercise are already introduced. There is a direct relationship between exercise intensity (external work) and the body's demand for oxygen. This oxygen demand is met by increasing pulmonary oxygen uptake (VO2). The cardiovascular system is responsible for transporting oxygen-rich blood from the lungs to the skeletal muscles, a process quantified as cardiac output (liters per minute) (Baggish and Wood, 2011).

Early investigations in 1899 by Henschen and skidwettlauf documented cardiac enlargement and bradyarrhythmias in individuals with above-normal exercise capacity without attendant signs of 
cardiovascular disease. Since that time, scientific understanding of the association between sport participation and specific cardiac abnormalities has paralleled advances in cardiovascular diagnostic techniques lead to athletic heart phenomenon. It is now well established that repetitive participation in vigorous physical exercise results in significant changes in myocardial structure and function. Recent increases in the popularity of recreational exercise and competitive athletics have led to a growing number of individuals exhibiting this phenomenon (Wilhelm and Seiler, 2012).

There is an ongoing debate about the true nature of the athlete's heart, whether it is only a physiological adaptation or a potentially pathological condition, fueled by reports about elevated biomarkers after prolonged endurance exercise. Abnormal ECG patterns are common in trained athletes and have been attributed to the physiologic cardiac adaptations and an elevated vagal tone that occur as a consequence of regular physical exercise. The frequency with which these ECG patterns occur is highly dependent on the sporting discipline, intensity and level of training, and also on gender and ethnicity (Pelliccia et al., 2000).

Phidippides cardiomyopathy also is another well-defined phenomenon in endurance athletes. It is developed because endurance sports call for a sustained increase in cardiac output for several hours, the heart is put into a state of volume overload. It has been shown that approximately one-third of marathon runners experience dilation of the right atrium and ventricle, have elevations of cardiac troponin and natriuretic peptides, and in a smaller fraction later develop small patches of cardiac fibrosis that are the likely substrate for ventricular tachyarrhythmias and sudden death (Trivax and McCullough, 2012). .

Age, gender, ethnicity and sporting discipline have a substantial impact on the magnitude of cardiac remodeling. Black athletes in particular exhibit striking abnormalities and left ventricular (LV) hypertrophy which may be regarded as an ethnic variant of the athlete's heart (Weinstock and Estes, 2013). Sport is thought to be a trigger and not a cause for life-threatening arrhythmia's in athletes with underlying cardiovascular diseases. A total of $14.4 \%$ of athletes showed distinct abnormalities, most commonly in cyclists, followed by cross country skiers and tennis players (Corrado et al., 2010). Compared to Caucasian athletes, ECG abnormalities and especially deep $\mathrm{T}$ wave inversions are more common in black athletes (Magalski et al., 2008; Papadakis et al., $2011 \&$ Rawlins et al., 2010).

Early studies considered athletic heart as a pathological changes due to strenuous training and thereby leads to progressive deterioration in function. These findings are characterized by chamber enlargement and ventricular hypertrophy with electric abnormalities including increased QRS voltage, abnormal $\mathrm{Q}$ waves, and $\mathrm{T}$-wave inversions (Maron and Pelliccia, 2006 \& Baggish and Wood, 2011). Currently, the athletic heart is regarded as a physiological increase in cardiac mass with circulatory and morphological adaptation to athletic training. Contemporary evidence supports the notion that this represents adaptive physiology, not preclinical disease.

Despite considerable advances in diagnostic tests, significant challenges remain in differentiating the athlete's heart from some types of cardiac disease. ECG and morphological changes found in athletes can mimic findings of hypertrophic 
cardiomyopathy, dilated cardiomyopathy, arrhythmogenic right ventricular (RV) cardiomyopathy (ARVC), and other cardiovascular diseases. The absence of a definitive diagnostic test or gold standard for many cardiovascular commonly results in diagnostic uncertainty (Weinstock and Estes 2013 \& Link and Estes, 2012). All these investigations depend upon heart dimensions and ECG or echocardiography findings but there is no biochemical markers used for prediction of the ongoing prognosis of this enlarged hearts.

Recently, there are many markers associated with immune-inflammatory and neurohormonal responses to heart damage have been identified. Among immunity and inflammatory markers, Neurohormones include Brain Natriuretic peptides derivatives (ProBNP), Cardiac injury (necrosis, apoptosis) markers including Cardiac troponin (cTnI and cTnT) and Matrix remodeling, endothelial dysfunction, and inflammatory markers including Galactic 3 share the ability to define the severity of the ongoing ventricular remodeling process (Emdin et al., 2009).

The aim of this study is to investigate athletic heart in adult long distance runners compared to young long distance runners using the biochemical heart failure markers including ProBNP, cTnI, and Galectin 3.

\section{Material and Methods:}

\section{Subjects:}

Two separated groups constituted subjects of this study. First group consisted of eight adult elite athletes completed cross country running experiment qualifying for Africa competition held in Al Gizera youth centre on Friday 24/2/2012. They completed a 12 $\mathrm{km}$. running. Their age were $24.7 \pm 1.2$ years, weight $68.5 \pm 1.9 \mathrm{~kg}$ and height $174.3 \pm 8.2$ $\mathrm{cm}$. The second group was eight subjects from the same experiment but their age was under 18 years and ran for eight $\mathrm{km}$. according to the protocol and ethics introduced by the Egyptian Federation of track and field events. Their age were $17.7 \pm$ 0.8 year, weight $62.4 \pm 2.2 \mathrm{~kg}$ and height $165.9 \pm 3.6 \mathrm{~cm}$. All subjects voluntarily provided written informed consent before participating. There was no clinical or analytical evidence for any disease among the participants.

\section{Blood analysis:}

Blood samples at rest were collected under aseptic conditions from the anti-cubital vein. Post-exercise blood sampling was performed immediately after the competition Samples were collected in tubes containing coagulation activator in resting for at least 30 minutes and immediately after competition, centrifuged and kept at $-20 \mathrm{C}^{\mathrm{o}}$ until tested. ProBNP, cTnI, and Galectin 3 levels were determined using a commercial ELISA kits provided by Teco medical group, Germany.

\section{Statistical analysis:}

All data are reported as mean \pm standard deviation (SD). The SPSS 17 software computer program was used to execute all statistical analyses. The non-parametric two related sample test ( $\mathrm{Z}$ test) was used to compare the means of each group before and after the training program. All of the data were correlated to exercise. The level of significance was set at $\mathrm{p} \leq 0.05$.

athlete's Z-tests were performed to assess differences between athletes completed cross country running group and athletes completed ran eight $\mathrm{km}$. Paired Z-tests were used to examine the difference between (prevs post-competition for the two groups and 
follow-up measures in each group \& in resting for at least 30 minutes immediately after competition measures in ProBNP, cTnI, and Galectin 3 levels. The non-parametric two related sample test ( $\mathrm{Z}$ test) were used to examine the main effects of competition \& 18 years ran eight $\mathrm{km}$. Statistical analyses were performed using the SPSS software (version 17.0, Chicago, IL) significance was set at $\mathrm{p} \leq 0.05$.
Results:

Table (1) summarizes results of the adult group pre and post exercise including the cardiac necrosis markers. Results revealed significant difference in all parameters investigated.

Table (1)

Results of adult group at pre-competition compared to post-competition

\begin{tabular}{|c|c|c|c|c|c|}
\hline & Pre- competition & $\begin{array}{c}\text { Post- } \\
\text { competition }\end{array}$ & $\mathrm{Z}$ & $\mathrm{P}$ & Significance \\
\hline & \multicolumn{2}{|c|}{ Mean \pm SD } & & & \\
\hline proBNP $(\mathrm{pg} / \mathrm{ml})$ & $281.25 \pm 62.16$ & $377.5 \pm 73.19$ & -2.521 & .012 & $\mathrm{~S}$ \\
\hline Galectin 3 (ng/ml) & $8.17 \pm 1.76$ & $14.58 \pm 2.27$ & -2.521 & .012 & $\mathrm{~S}$ \\
\hline cTnI $(\mathrm{pg} / \mathrm{ml})$ & $9.31 \pm 2.71$ & $22.05 \pm 5.93$ & -2.521 & .012 & $\mathrm{~S}$ \\
\hline
\end{tabular}

Values are means $\pm \mathrm{SD}$; $p$ values obtained from $\mathrm{Z}$-tests

Significantly different from 2 athletes groups, p, 0:05

Table (2)

Results of under 18 years group at rest compared to after exercise

\begin{tabular}{|c|c|c|c|c|c|}
\hline & \multicolumn{1}{|c|}{ Pre- competition } & $\begin{array}{c}\text { Post- } \\
\text { competition }\end{array}$ & $\mathrm{Z}$ & P & Significant \\
\hline & \multicolumn{2}{|c|}{ Mean \pm SD } & & & \\
\hline proBNP (pg/ml) & $271 \pm 57.79$ & $336.25 \pm 43.85$ & -2.380 & .017 & S \\
\hline Galectin 3 (ng/ml) & $6.82 \pm 0.91$ & $12.512 \pm 2.02$ & -2.521 & .012 & S \\
\hline cTnI (pg/ml) & $7.27 \pm 1.79$ & $18.56 \pm 3.92$ & -2.521 & .012 & S \\
\hline
\end{tabular}

Table (2) showed the same findings resulted in the adult group for under 18 years' group. All investigated parameters were also significant with higher levels post-exercise 
Table (3)

Results of adult group compared to under 18 years at rest (pre-exercise)

\begin{tabular}{|c|c|c|c|c|c|}
\hline & \multicolumn{2}{|c|}{ Mean \pm SD } & Z & P & Significant \\
\hline & Adult & Under 18 years & & & \\
\hline proBNP $(\mathrm{pg} / \mathrm{ml})$ & $281.25 \pm 62.16$ & $271 \pm 57.79$ & -.420 & .674 & NS \\
\hline Galectin 3 (ng/ml) & $8.17 \pm 1.76$ & $6.82 \pm 0.91$ & -2.100 & .036 & $\mathrm{~S}$ \\
\hline cTnI (pg/ml) & $9.31 \pm 2.71$ & $7.27 \pm 1.79$ & -1.960 & .050 & $\mathrm{~S}$ \\
\hline
\end{tabular}

(Table 3) showed that when the two groups are compared at pre-competition, result revealed significant high levels in adult group in galectin 3 and cardiac troponin I (cTnI) but no differences in N-terminal pro-brain natriuretic peptide (proBNP) levels.

Table (4)

Results of adult group compared to under 18 years post-exercise

\begin{tabular}{|c|c|c|c|c|c|}
\hline & \multicolumn{2}{|c|}{ Mean \pm SD } & Z & P & Significant \\
\hline & Adult & Under 18 years & & & \\
\hline proBNP $(\mathrm{pg} / \mathrm{ml})$ & $377.5 \pm 73.19$ & $336.25 \pm 43.85$ & -1.540 & .123 & NS \\
\hline Galectin 3 (ng/ml) & $14.58 \pm 2.27$ & $12.512 \pm 2.02$ & -1.540 & .123 & NS \\
\hline cTnI $(\mathrm{pg} / \mathrm{ml})$ & $22.05 \pm 5.93$ & $18.56 \pm 3.92$ & -1.540 & .123 & NS \\
\hline
\end{tabular}

Table (4) showed the most important results were postulated in which revealed no differences in response to exercise in both groups. There are elevated post-exercise levels in all three cardiac parameters regardless to age.

There are a positive correlation between competition and proBNP $(r=909, p<0.001)$ and between proBNP and cTnI ( $\mathrm{r}=732$, $\mathrm{p}<0.04)$ in adult group. In under 18 years group run $8 \mathrm{KM}$, the correlation were found between galectin 3 and proBNP either before and after exercise.

\section{Discussion:}

The risk of myocardial damage by endurance competition is under debate because of reports on exercise-associated increases in cardiac biomarkers and myocardial dysfunction (Scharhag et al., 2008). Aim of this study was to investigate athletic heart phenomenon presented in many athletes especially who participated in long heavy endurance sports like long distance and marathon runners.

All cardiac parameters were elevated postexercise compared with pre-exercise levels. These results indicated at least minor cardiac necrosis due to heavy exercise sessions. Moreover, rest cardiac markers' levels in adult were higher than that observed in younger athletes (under 18 years) indicating chronic effect of such sports with the recurrent participation in.

Release of cardiac bio-markers were observed in a previous study by Fortescue et al. (2007) including myoglobin, cardiac troponin-I, creatine kinase, and creatine 
kinase myocardial band, and B-type natriuretic peptide. Also, volume depletion with reduced renal filtration occurs with elevated blood urea nitrogen, serum creatinine, and cystitis $\mathrm{C}$ were observed by McCullough et al. (2011). Pelliccia et al., 2010 suggested that increases in cardiac troponin are only mild in athletes and of short duration and therefore, may reflect a reversible membrane leakage of cardiomyocytes with troponin release from the free cytosolic pool.

Hättasch et al. (2013) found pre-exercise plasma levels of galectin-3 were higher in long distance runners than control active non-athletic groups. Also plasma galectin-3 in endurance athletes increased further from pre to post- competition $(12.8 \pm 3.4 \mathrm{ng} / \mathrm{mL}$ to $19.9 \pm 3.9 \mathrm{ng} / \mathrm{mL}, \mathrm{p}<0.001)$. They concluded that Plasma galectin-3 is substantially elevated in endurance athletes, both after short-term abstinence and especially after running. The galectin-3 levels do not correlate with cardiac function or other biomarkers. galectin-3 increase during endurance exercise may originates primarily from skeletal muscle.

NT-proBNP concentrations under resting conditions are not elevated in healthy athletes with or without signs of the athlete's heart. Short term elevations of NT-proBNP after prolonged exercise may have cyto-protective and growth-regulating effects on the athlete's heart (Scharhag et al., 2008). However, studies on persistent RV dysfunction in highly trained endurance athletes with ventricular arrhythmias suggest that elevated cardiac bio-markers may reflect an exerciseinduced myocardial injury to the RV (Heidbuchel et al., 2003 \& Ector et al., 2007).

Post-marathon (Boston marathon 2006) cardiac markers were elevated in at least $40 \%$ of participants with Troponin $\mathrm{T}$ level above normal and N-terminal pro-brain natriuretic peptide (NT-proBNP) increased significantly after the race. The increase in bio-markers correlated with post-race diastolic dysfunction, increased pulmonary pressures, right ventricular dysfunction, and inversely with training mileage. Athletes with the lowest training mileages exhibited the highest risk for myocardial injury and dysfunction (Neilan et al., 2006 (A)). At least one-third of individuals after a marathon, irrespective of baseline factors, speed, or conditioning, will have a rise and fall of cardiac troponin and B-type natriuretic peptide (Trivax et al., 2010).

La Gerche et al. (2008) postulated that - in all cases - the heart faces increased pressure and volume overload and responds by increasing left ventricular chamber size, thickness, and mass. Running for long distances initiates cardiac injury, including increased release of catecholamines and resultant coronary vasoconstriction, increased heart rate leading to decreased diastolic filling time of the coronary arteries, increased demand for oxygen, changes in free fatty acid metabolism, lactic acidosis, and metabolic derangements (Goodman et al., 2013). As a result the heart is unable to keep up with the demands of running and increasing right heart preload and afterload, and begins to dilate and stretch in response to these hemodynamic changes (Krip et al., 1997). These changes may be more pronounced in those with less training (Neilan et al., 2006 (B)).

Right heart dilation and hypokinesis after prolonged strenuous exercise has been observed using cardiac magnetic resonance imaging (Trivax et al., 2010 \& Breuckmann et al., 2009). Diastolic dysfunction is frequently observed in both younger and 
older athletes (Knebel et al., 2009). After one month follow-up of marathon race, systolic function of the $\mathrm{LV}$ and $\mathrm{RV}$ returned to normal, while LV and RV diastolic function were still abnormal (Neilan et al., 2006 (A)). However, to date there are no reports of a higher incidence of heart failure in athletes (Wilhelm and Seiler, 2012).

During the recovery period after long distance run, the cardiac geometric dimensions are restored, and many athletes continue this cycle with training, running, cardiac disturbances, and again cardiac recovery. With this repetitive stretch of the chambers and restoration of the chamber geometry, we believe there are individuals prone to developing chronic structural changes, including chamber dilation and myocardial fibrosis, as a response to the repetitive volume overload and dynamic strain of the heart (Mousavi et al., 2009 \& Gerche et al., 2012).

In Conclusion, it is unclear whether long distance runners group, in particular, will go on to develop patches of cardiac fibrosis. These changes are asymptomatic and probably occur over many years. However, in those who experience ventricular arrhythmias and/or sudden cardiac death there is this lethal disorder, Phidippides cardiomyopathy. Thus, it is rational to develop risk prediction and screening methods for this disorder to allow counseling for individuals who are considering endurance sports such as cross country and marathon running.

\section{Refrences:}

1. Baggish, AL and Wood, MJ (2011): Athlete's Heart and Cardiovascular Care of the Athlete : Scientific and Clinical Update Circulation, 2011; 123: 2723-2735
2. Breuckmann F, M“ohlenkamp S, Nassenstein K, et al. (2009): Myocardial late gadolinium enhancement: prevalence, pattern, and prognostic relevance in marathon runners. Radiology. 2009;251:50-57.

3. Ector J, Ganame J, van der Merwe N, Adriaenssens B, Pison L, Willems R, et al. (2007): Reduced right ventricular ejection fraction in endurance athletes presenting with ventricular arrhythmias: a quantitative angiographic assessment. Eur Heart J. 2007;28(3):345-53.

4. Emdin M, Vittorini S, Passino C, Clerico A (2009): Old and new biomarkers of heart failure European Journal of Heart Failure (2009) 11, 331-335

5. Fortescue EB, Shin AY, Greenes DS, et al. (2007): Cardiac troponin increases among runners in the Boston Marathon. Ann Emerg Med. 2007;49:137-143.

6. Gerche A, Burns AT, Mooney DJ, Inder WJ, Taylor AT, Bogaert J, MacIsaac AI, Heidbüchel H, Prior DL (2012): Exerciseinduced right ventricular dysfunction and structural remodelling in endurance athletes Eur Heart J (2012) 33 (8): 998-1006.

7. Goodman J, Thomas S and Burr JF (2013): Cardiovascular risks of physical activity in apparently healthy individuals Risk evaluation for exercise clearance and prescription Canadian Family Physician 59: 46 - 49

8. Hättasch $\mathbf{R}$, Spethmann $\mathbf{S}$, de Boer $\mathbf{R}$, Ruifrok WP, Schroeckh S, Schimke I, Wagner M, Durmus T, Borges AC, Sanad W, Baumann G, Knebel F (2013): Possible mechanism of Galectin-3 increase in endurance athletes Clin Res Cardiol 102, Suppl 1, April 2013, P1837

9. Heidbuchel H, Hoogsteen J, Fagard R, Vanhees L, Ector H, Willems R, et al. (2003): High prevalence of right ventricular involvement in endurance athletes with ventricular arrhythmias. Role of an electrophysiologic study in risk stratification. Eur Heart J. 2003;24(16):1473-80.

10. Henschen S. Skidlauf und skidwettlauf (1899): eine medizinische sportstudie. Mitt Med Klin Upsala.1899:2. 
11. Knebel F, Schimke I, Schroeckh S, et al. (2009): Myocardial function in older male amateur marathon runners: assessment by tissue Doppler echocardiography, speckle tracking, and cardiac biomarkers. J Am Soc Echocardiogr. 2009;22:803-809.

12. Krip B, Gledhill N, Jamnik V, et al. (1997): Effect of alterations in blood volume on cardiac function during maximal exercise. Med Sci Sports Exerc. 1997;29:1469-1476.

13. La Gerche A, Connelly KA, Mooney DJ. (2008): Biochemical and functional abnormalities of left and right ventricular function after ultra-endurance exercise. Heart. 2008;94:860-866.

14. Link MS, Estes NAM III. (2012): Sudden cardiac death in the athlete: bridging the gaps between evidence, policy and practice. Circulation. 2012;125:2511-2516.

15. Magalski A, Maron BJ, Main ML, McCoy M, Florez A, Reid KJ, et al. (2008): Relation of race to electrocardiographic patterns in elite American football players. J Am Coll Cardiol. 2008;51(23):2250-5.

16. Maron BJ, Pelliccia A. (2006): The heart of trained athletes: cardiac remodeling and the risks of sports, including sudden death. Circulation. 2006;114:1633-1644.

17. McCullough PA, Chinnaiyan KM, Gallagher MJ, et al. (2011): Changes in renal markers and acute kidney injury after marathon running.Nephrology (Carlton). 2011;16:194199.

18. Mousavi N, Czarneck A, Kumar K, et al. (2009): Relation of biomarkers and cardiac magnetic resonance imaging after marathon running.Am J Cardiol. 2009;103:1467-1472.

19. Neilan TG, Januzzi JL, Lee-Lewandrowski E, Ton-Nu TT, Yoerger DM, Jassal DS, et al. (2006) (A): Myocardial injury and ventricular dysfunction related to training levels among nonelite participants in the Boston marathon.Circulation. 2006;114(22):2325-33.

20. Neilan TG, Yoerger DM, Douglas PS, Marshall JE, Halpern EF, Lawlor D, et al. (2006) (B): Persistent and reversible cardiac dysfunction among amateur marathon runners. Eur Heart J. 2006;27(9):1079-84.

21. Papadakis M, Carre F, Kervio G, Rawlins J, Panoulas VF, Chandra N, et al. (2011): The prevalence, distribution, and clinical outcomes of electrocardiographic repolarization patterns in male athletes of African/Afro-Caribbean origin. Eur Heart J. 2011; 32: .

22. Pelliccia A, Maron BJ, Culasso F, Di Paolo FM, Spataro A, Biffi A, et al. (2000): Clinical significance of abnormal electrocardiographic patterns in trained athletes. Circulation. 2000;102(3):278-84.

23. Pelliccia A, Kinoshita N, Pisicchio C, Quattrini F, Dipaolo FM, Ciardo R, et al. (2010): Long-term clinical consequences of intense, uninterrupted endurance training in olympic athletes. J Am Coll Cardiol. 2010;55(15): 1619-25.

24. Rawlins J, Carre F, Kervio G, Papadakis M, Chandra N, Edwards C, et al. (2010): Ethnic differences in physiological cardiac adaptation to intense physical exercise in highly trained female athletes. Circulation. 2010; 121(9):1078-85.

25. Scharhag J, George K, Shave R, Urhausen A, Kindermann W. (2008): Exercise associated increases in cardiac biomarkers. Med Sci Sports Exerc. 2008;40(8):1408-15.

26. Trivax JE, Franklin BA, Goldstein JA, et al. (2010): Acute cardiac effects of marathon running. J Appl Physiol. 2010;108:1148-1153

27. Trivax JE and McCullough PA (2012): Phidippides Cardiomyopathy: A Review and Case Illustration Clin. Cardiol. 35, 2, 69-73 (2012)

28. Weinstock J and Estes NA (2013): The Heart of an Athlete Black, White, and Shades of Grey with No Gold Standard Circulation. 2013;127:1757-1759.

29. Wilhelm, MW and Seiler, C (2012): The athlete's heart: different training responses, gender and ethnicity dependencies Cardiovascular Medicine 2012; 15(3):69-78 
